\title{
PAPER \\ Quantum Associative Memory with Quantum Neural Network via Adiabatic Hamiltonian Evolution
}

\author{
Yoshihiro OSAKABE ${ }^{\dagger a)}$, Student Member, Hisanao AKIMA ${ }^{\dagger}$, Member, $^{\text {Masao SAKURABA }}{ }^{\dagger}$, Nonmember, \\ Mitsunaga KINJO ${ }^{\dagger \dagger}$, Member, and Shigeo SATO ${ }^{\dagger}$, Senior Member
}

\begin{abstract}
SUMMARY There is increasing interest in quantum computing, because of its enormous computing potential. A small number of powerful quantum algorithms have been proposed to date; however, the development of new quantum algorithms for practical use remains essential. Parallel computing with a neural network has successfully realized certain unique functions such as learning and recognition; therefore, the introduction of certain neural computing techniques into quantum computing to enlarge the quantum computing application field is worthwhile. In this paper, a novel quantum associative memory (QuAM) is proposed, which is achieved with a quantum neural network by employing adiabatic Hamiltonian evolution. The memorization and retrieval procedures are inspired by the concept of associative memory realized with an artificial neural network. To study the detailed dynamics of our QuAM, we examine two types of Hamiltonians for pattern memorization. The first is a Hamiltonian having diagonal elements, which is known as an Ising Hamiltonian and which is similar to the cost function of a Hopfield network. The second is a Hamiltonian having non-diagonal elements, which is known as a neuro-inspired Hamiltonian and which is based on interactions between qubits. Numerical simulations indicate that the proposed methods for pattern memorization and retrieval work well with both types of Hamiltonians. Further, both Hamiltonians yield almost identical performance, although their retrieval properties differ. The QuAM exhibits new and unique features, such as a large memory capacity, which differs from a conventional neural associative memory. key words: associative memory, quantum computing, adiabatic Hamiltonian evolution, neural network
\end{abstract}

\section{Introduction}

Quantum computing has attracted considerable research interest, because of its inherent parallel computing characteristic, which is achieved using superposition states. For realization of a quantum computer, reliable hardware and software must be developed. As a result of recent progress in nanotechnology, the implementation of a quantum computer has become a more realistic prospect. In fact, Dwave Systems Inc. has developed a quantum processor composed of 512 qubits [1], [2], and this achievement could be a breakthrough for the development of quantum computing architectures. Hence, quantum annealing (QA) [3], which is the quantum version of simulated annealing [4] and

Manuscript received April 22, 2017.

Manuscript revised July 12, 2017.

Manuscript publicized August 9, 2017.

$\dagger$ The authors are with Laboratory for Nanoelectronics and Spintronics, Research Institute of Electrical Communication, Tohoku University, Sendai-shi, 980-8577 Japan.

${ }^{\dagger \dagger}$ The author is with Department of Electrical and Electronics Engineering, University of the Ryukyus, Okinawa-ken, 903-0213 Japan.

a) E-mail: osakabe@ riec.tohoku.ac.jp DOI: $10.1587 /$ transinf.2017EDP7138 is realized via adiabatic Hamiltonian evolution, has been demonstrated, although the range of application was restricted [5]. On the other hand, the development of new algorithms is still necessary for practical applications, despite the fact that a small number of powerful quantum algorithms have been proposed [6], [7]. Recently, neural computing techniques have successfully provided conventional computers with an effective means of acquiring new algorithms through learning [8]. It is worth considering the fusion of these two fields to enlarge the application fields of quantum computers. In fact, some pioneers have investigated the quantum analog of neural networks. Since Kak first proposed a quantum neural network (QNN) model in 1995 [9], various QNN models have been proposed (see the reviews given in Refs. [10], [11]). Quantum-inspired evolutionary algorithms have been investigated, and the concept and principles of quantum computing, such as a qubit and the superposition of states, have been incorporated into neural computing [12], [13]. Contrary to these successful proposals, which have contributed to the improvement of neural networks, Sato et al. have introduced the concept of neural computing to quantum computing [14]. These researchers have introduced the quantum dynamics of adiabatic Hamiltonian evolution [15] with a QNN to neural computing and solved combinatorial optimization problems; this approach is called neuromorphic adiabatic quantum computation (NAQC) [14], [16]. Further, Osakabe et al. have followed the analogy between a QNN and a neural network and proposed a quantum learning method for a QNN by imitating the Hebb rule [17]. Similar to these studies, several methods to introduce neural functions into quantum computing have also been explored. Ventura et al. have introduced the association function, which is a fundamental function of neural networks, to quantum computing, and proposed a quantum associative memory (QuAM) [18]. These researchers have used quantum logic gates to memorize patterns and modified Grover's database search algorithm [7] to recall memorized patterns. Peruš et al. have reported that the mathematical formalisms of an associative neural network model and quantum theory are analogous [19]. They have composed a QNN and implemented a quantum content addressable memory. Further, Allauddin et al. have incorporated the cytoskeleton of a biological neuron into a QNN and trained the QNN to implement a content addressable memory [20].

Taking into account the hardware compatibility of adi- 
abatic Hamiltonian evolution, which is demonstrated by D-wave Systems Inc., this paper proposes a novel QuAM model realized on a QNN, the dynamics of which is based on QA and NAQC. In the following, we explain some of the fundamental theory of our proposed QuAM model and discuss its memorization and retrieval procedures. To investigate the detailed dynamics of our QuAM, we examine the performance of two Hamiltonians in the context of multiple pattern recognition. Base on presented numerical simulation results, we evaluate the QuAM performance.

\section{Basic Theory}

In this section, we explain adiabatic Hamiltonian evolution, which is utilized in the dynamics of our QuAM model.

\subsection{Quantum Annealing}

The first formulation shown in Eq. (1) was proposed by Kadowaki et al. in 1998 [3], to solve combinatorial optimization problems such as determining the ground state of a spin glass. This method is known as QA and the adiabatic time evolution of its Hamiltonian is defined as

$$
H(t)=\Gamma(t) H_{\text {tun }}+H_{\text {fin }},
$$

where $H_{\text {tun }}$ and $H_{\text {fin }}$ denote the tunnel and final Hamiltonians, respectively. $H_{\text {tun }}$ allows state changes via tunneling and yields faster state transition to global minima. $H_{\text {fin }}$ is chosen according to the desired operation or target problem. Because $\Gamma(t)$ is a decreasing function in time, the effect of $H_{\text {tun }}$ is reduced with increasing $t$. Finally, the quantum state converges to a ground state of $H_{\text {fin }}$.

\subsection{Adiabatic Quantum Computation}

Farhi et al. proposed another formulation of adiabatic Hamiltonian evolution, which is shown in Eq. (2) and which has been used to solve the 3-satisfiability (3-SAT) problem [15]. This algorithm is known as adiabatic quantum computing (AQC) and its computing performance is considered to be closely related to that of QA. The time evolution of the Hamiltonian is given as

$$
H(t)=\left(1-\frac{t}{T}\right) H_{\text {init }}+\frac{t}{T} H_{\text {fin }},
$$

where $T$ denotes the period of the Hamiltonian evolution. The initial Hamiltonian $H_{\text {init }}$ is given as a superposition of all states, and the final Hamiltonian $H_{\text {fin }}$ includes solution states. The initial state $|\Psi(0)\rangle$ is given as the ground state of $H_{\text {init }}$, which includes all possible states, such that

$$
|\Psi(0)\rangle=\frac{1}{\sqrt{2^{N}}} \sum_{z=0}^{2^{N}-1}|z\rangle,
$$

where $N$ is the number of qubits. $|z\rangle$ is the decimal notation of the quantum system and is expressed as

$$
|z\rangle=\left|x_{N}\right\rangle \otimes \cdots \otimes\left|x_{2}\right\rangle \otimes\left|x_{1}\right\rangle=\left|x_{N} \cdots x_{2} x_{1}\right\rangle,
$$

where $\left|x_{i}\right\rangle$ denotes a single qubit state and takes $|0\rangle$ or $|1\rangle$ exclusively. If a sufficiently large $T$ is chosen, the quantum system evolves adiabatically, and the adiabatic theorem [21] indicates that the quantum system state remains near the ground state during the evolution. Along with increasing $t$, non-desired states vanish gradually. Finally, desired states as optimal solutions given by $H_{\text {fin }}$ can be found by measuring $|\Psi(T)\rangle$. However, if any energy level crossing occurs during the evolution, successful operation is not guaranteed.

\subsection{Neuromorphic Adiabatic Quantum Computation}

Sato et al. introduced a neuro-inspired approach to AQC, namely, NAQC [14], [16], and proposed a new method of generating $H_{\text {fin }}$.

In order to clarify the relation between a qubit and a neuron, let us consider a $1 / 2$-spin system as sample quantum system, where $|0\rangle$ and $|1\rangle$ correspond to a down- and up-spin, respectively. The Hamiltonian of this system is written as

$$
H=-\frac{1}{2} \sum_{i} \sum_{j} J_{i j} \sigma_{i} \cdot \sigma_{j}
$$

where $J_{i j}$ is the magnitude of the spin-spin interaction between the $i$-th and $j$-th $1 / 2$-spins, and $\sigma_{i}$ and $\sigma_{j}$ denote the Pauli spin matrices. The network behavior is determined by this Hamiltonian, which is called a Heisenberg Hamiltonian. This is similar to the fact that the behavior of the Hopfield network [22] is given by the energy function, such that

$$
E=-\frac{1}{2} \sum_{i} \sum_{j} w_{i j} o_{i} o_{j}
$$

where $w_{i j}$ is the synaptic weight between the $i$-th and $j$-th neurons and $o_{i}$ and $o_{j}$ are neuron outputs. Inspired by this analogy, one can introduce quantum mechanical dynamics to the Hopfield network. By comparing Eqs. (5) and (6), the synaptic weight matrix is converted into a Hamiltonian, which corresponds to $H_{\text {fin }}$ in Eq. (2). Thus, it becomes possible to relate a neural network with a QNN. The conversion method is described in detail in Sect. 3.1.2.

\section{Model of Quantum Associative Memory}

\subsection{Memorization Procedure}

Let us assume that the QuAM model composed of $N$ qubits memorizes a set $\mathcal{S}$ of $M$ patterns. The patterns are $N$ dimensional random vectors, the elements of which take \pm 1 randomly. One of the vectors is denoted by $\xi_{\mu}=$ $\left(y_{1}, y_{2}, \cdots, y_{N}\right)^{\mathrm{T}}$, where $\mu$ is the pattern index $(1 \leq \mu \leq M)$. $y_{i}$ satisfies

$$
y_{i}=2 x_{i}-1
$$

where $i$ is the index of qubits, for calculation convenience. 
The QuAM memorizes a pair comprised of an original pattern and its inverted pattern $\left(\xi_{\mu}\right.$ and $\bar{\xi}_{\mu}$, respectively) with equal probability, which is also true for the Hopfield network. Thus, the maximum of $M$ is given as $M_{\max }=2^{N-1}$. In a conventional neural network, a pattern is memorized with an autocorrelation matrix regarded as a synaptic weight matrix. Therefore, we relate an autocorrelation matrix of memorized patterns with a Hamiltonian. To study the detailed dynamics of the QuAM, we examine two types of Hamiltonians for pattern memorization $H_{\text {mem }}$; one is based on QA and the other is based on NAQC.

\subsection{1 $H_{\text {mem }}$ Based on Ising Hamiltonian (QA)}

The first method of composing $H_{\mathrm{mem}}$ is based on QA. Thus, we formulate this expression by following the Hamiltonian for the database search problem [23] and obtain

$$
H_{\mathrm{mem}}^{\mathrm{IS}}=I^{\left(2^{N}\right)}-\sum_{\mu}\left|z_{\mu}\right\rangle\left\langle z_{\mu}\right|
$$

where $\left|z_{\mu}\right\rangle$ is the decimal notation of $\xi_{\mu}$, and $I^{\left(2^{N}\right)}$ is the $2^{N} \times$ $2^{N}$ identity matrix. In this formulation, $H_{\mathrm{mem}}^{\mathrm{IS}}$ has diagonal elements only. Because $H_{\mathrm{mem}}^{\mathrm{IS}}$ is similar to the cost function of a Hopfield network, it is confirmed that the $H_{\text {mem }}^{\mathrm{IS}}$ ground states are given as superposition states of all patterns in $\mathcal{S}$. Thus, the total probability $P_{\text {total }}^{\mathrm{IS}}$ of all memorized patterns (i.e. the $\xi_{\mu}$ s) always takes its maximum (50\%), as explained in the next section.

\subsection{2 $H_{\text {mem }}$ Based on Neuro-Inspired Hamiltonian (NAQC)}

The other method of composing $H_{\text {mem }}$ follows NAQC. Here, a neuro-inspired Hamiltonian $H_{\mathrm{mem}}^{\mathrm{NI}}$ for pattern memorization is converted from the autocorrelation matrix of the patterns in $\mathcal{S}$. The autocorrelation matrix $W$ for the QuAM is defined as

$$
W=\frac{1}{N}\left(\sum_{\mu=1}^{M} \xi_{\mu} \xi_{\mu}^{\mathrm{T}}-M I^{(N)}\right) .
$$

We follow the method proposed in [14], which enables us to convert neuron-neuron interactions into qubit-qubit interactions, and define $H_{\mathrm{mem}}^{\mathrm{NI}}$ based on these interactions, such that

$$
H_{\mathrm{mem}}^{\mathrm{NI}}=\lambda I^{\left(2^{N}\right)}+\sum_{i} \sum_{j<i}\left|w_{i j}\right|\left\{H_{i j}\left(w_{i j}\right)+H_{i j}^{\dagger}\left(w_{i j}\right)\right\},
$$

where $\lambda$ is a constant. $H_{i j}\left(w_{i j}\right)$, which denotes the interaction between the $i$-th and $j$-th qubits, is given as

$$
\begin{aligned}
& H_{i j}\left(w_{i j}\right) \\
& = \begin{cases}(I^{(2)} \otimes \cdots \overbrace{\otimes a \otimes}^{i-t h} \cdots \overbrace{\otimes a \otimes}^{j-t h} \cdots \otimes I^{(2)}) & \left(w_{i j}>0\right), \\
O^{\left(2^{N}\right)} & \left(w_{i j}=0\right), \\
(I^{(2)} \otimes \cdots \overbrace{\otimes a \otimes}^{i-t h} \cdots \overbrace{\otimes a^{\dagger} \otimes}^{j-t h} \cdots \otimes I^{(2)}) & \left(w_{i j}<0\right),\end{cases}
\end{aligned}
$$

where $a$ and $a^{\dagger}$ denote the fermion annihilation and creation operators, respectively, and $O^{\left(2^{N}\right)}$ is the $2^{N} \times 2^{N}$ zero matrix. As explained in previous reports [14], [16], both excitatory $\left(w_{i j}>0\right)$ and inhibitory $\left(w_{i j}<0\right)$ interactions can be realized effectively.

In this formulation, $H_{\mathrm{mem}}^{\mathrm{NI}}$ has non-diagonal elements and differs from $H_{\mathrm{mem}}^{\mathrm{IS}}$. One of the aims of examining two types of Hamiltonians is evaluating the influence of the diagonal and non-diagonal elements in $H_{\text {mem }}$ on the QuAM dynamics. In contrast to $H_{\mathrm{mem}}^{\mathrm{IS}}$, it is not obvious that the ground state of $H_{\mathrm{mem}}^{\mathrm{NI}}$ can be given as a superposition state of the memorized patterns. In order to confirm the memorization capability of $H_{\mathrm{mem}}^{\mathrm{NI}}$, we conducted numerical simulations, for which a sufficiently large amount of $\mathcal{S}$ s were prepared. For each $\mathcal{S}$, we calculated the eigenstates of $H_{\mathrm{mem}}^{\mathrm{NI}}$ and obtained its ground state $\left|\Psi_{\mathrm{gs}}^{\mathrm{NI}}\right\rangle$. The total probability $P_{\text {total }}^{\mathrm{NI}}$ was calculated as $\sum_{\mu}\left|\left\langle z_{\mu} \mid \Psi_{\mathrm{gs}}^{\mathrm{NI}}\right\rangle\right|^{2}$. In other words, $P_{\text {total }}^{\mathrm{NI}}$ means the sum of the probabilities of the quantum states corresponding to the memorized patterns $\xi_{\mu} \mathrm{s}$. Because of the symmetric property of $W$, the QuAM cannot avoid memorizing inverted patterns $\bar{\xi}_{\mu}$. Hence, the maximum of $P_{\text {total }}^{\mathrm{NI}}$ must be $50 \%$ at most, following the same reasoning presented in Sect. 3.1.1. As shown in Fig. 1, $\left\langle P_{\text {total }}^{\mathrm{NI}}\right\rangle$, which is the average of $P_{\text {total }}^{\mathrm{NI}}$, increases with increasing $M$ and reaches $50 \%$ in the case of $2 \leq M \leq 5$. In comparison with the Hopfield network, this $M$-dependency seems strange at a glance. Theoretically, the sum of all synaptic weight matrices for $M_{\max }$ patterns is identical to the zero matrix. As a special case, the zero matrix gives the superposition of all possible states; hence, the QuAM memorizes all possible states simultaneously. This suggests that $\left\langle P_{\text {total }}^{\mathrm{NI}}\right\rangle$ for $N=6$ reaches $50 \%$ at $M=M_{\max }$. However, we cannot confirm such cases numerically, because our computer has insufficient memory (128 GB). Here, we found that the nominal memory capacity $M_{\text {cap }}$ of the QuAM is $M_{\max }=2^{N-1}$, because $\left\langle P_{\text {total }}^{\mathrm{NI}}\right\rangle$ takes its maximum, and confirmed that $H_{\mathrm{mem}}^{\mathrm{NI}}$ can store multiple patterns in its ground states. We should note that this estimation was calculated without executing an adiabatic Hamiltonian change. The actual retrieval per-

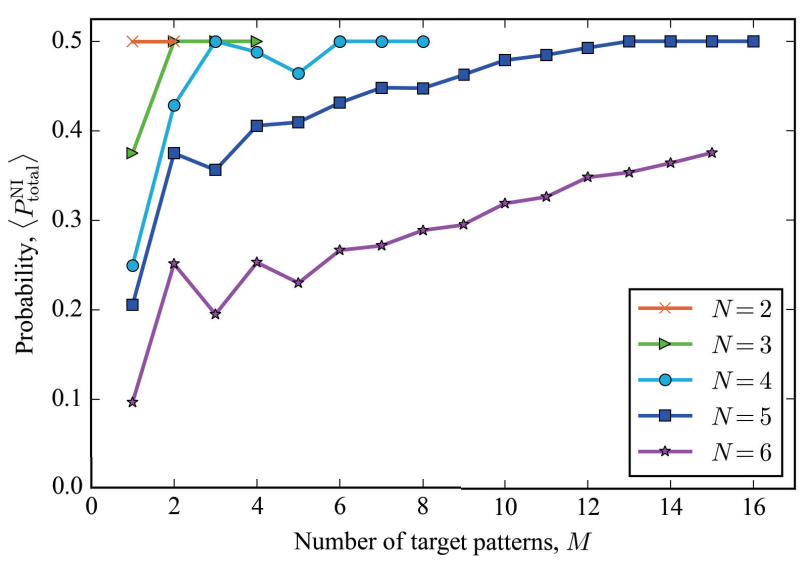

Fig. 1 Sum of nominal estimated probabilities of all memorized patterns with $H_{\mathrm{mem}}^{\mathrm{NI}},\left\langle P_{\text {total }}^{\mathrm{NI}}\right\rangle$. 
formance is discussed in Sect. 4.

\subsection{Retrieval Procedure}

\subsubsection{Associative Key Input}

A conventional neural associative memory converges to a certain state corresponding to one of the memorized patterns according to the initial state given as the key input, which is usually a degraded target pattern. On the other hand, the QuAM state is given as a superposition state and does not converge to a specific state unless some specific treatment is applied. Therefore, we apply an external Hamiltonian $H_{\text {fix }}$ to partially fix the state of some qubits, which imitates the role of the key input for a neural associative memory. First, we choose $\xi_{\mu}^{\prime}$ as a target pattern from the $\xi_{\mu}$ s and generate the key input $\xi_{\text {key }}$ by replacing one qubit in $\xi_{\mu}^{\prime}$ with an unfixed qubit. Thus, the retrieval process is regarded as recalling the original target pattern $\xi_{\mu}^{\prime}$ from $\xi_{\text {key }} . H_{\text {fix }}$ is given as

$$
\begin{aligned}
H_{\mathrm{fix}}= & \left(\kappa_{1} \otimes I^{(2)} \otimes \cdots I^{(2)}+I^{(2)} \otimes \kappa_{2} \otimes \cdots I^{(2)}\right. \\
& \left.+\cdots+I^{(2)} \otimes I^{(2)} \otimes \cdots \otimes \kappa_{N}\right) .
\end{aligned}
$$

$\kappa_{i}$ is defined as

$$
\kappa_{i}= \begin{cases}+\sigma^{z} & \left(\text { if } y_{i} \text { is }+1\right), \\ I^{(2)} & \left(\text { if } y_{i}\right. \text { is unfixed), } \\ -\sigma^{z} & \left(\text { if } y_{i} \text { is }-1\right),\end{cases}
$$

where $\sigma^{z}$ is the $z$-component of the Pauli spin matrices. When we add $H_{\text {fix }}$ during the Hamiltonian evolution, we can retrieve a target pattern according to $\xi_{\mathrm{key}}$.

\subsection{2 $H_{\text {fix }}$ Application Method}

We re-formulate Eqs. (1) and (2) in consideration of the $H_{\text {fix }}$ application. Equation (1) can be replaced with

$$
H(t)=\Gamma(t) H_{\mathrm{tun}}+\left(H_{\mathrm{mem}}^{\mathrm{IS}}+A_{\mathrm{fix}} H_{\mathrm{fix}}\right)
$$

where $A_{\text {fix }}$ is a scaling factor of $H_{\text {fix }} . H_{\text {tun }}$ is the tensor product of the $\sigma^{x}$ s. Equation (2) can be replaced with

$$
H(t)=\left(1-\frac{t}{T}\right) H_{\text {init }}+\frac{t}{T}\left(H_{\mathrm{mem}}^{\mathrm{NI}}+A_{\mathrm{fix}} H_{\mathrm{fix}}\right) .
$$

Note that the quantum state of a QNN also evolves with $t$ as

$$
|\Psi(t+\Delta t)\rangle=U(\Delta t)|\Psi(t)\rangle=e^{-\frac{i \mid H(t)+\Delta H \backslash \Delta t}{\hbar}}|\Psi(t)\rangle,
$$

where the operator $U(\Delta t)$ is given by the Padé approximation [24]. $\Delta H$ denotes a slight energy fluctuation and facilitates convergence of a QNN to its ground state; this approach has been proposed and verified by Kinjo et al. [16].

\subsection{Summary of QuAM Operations}

In this subsection, we summarize the memorization and retrieval operations of our QuAM model, as follows.

\section{<Memorization Procedure $>$}

1. A set $\mathcal{S}$ of memory patterns, i.e., $\xi_{\mu}$ s, is prepared.

2. The autocorrelation matrix $W$ is generated according to Eq. (9).

3. The memorization Hamiltonian $H_{\text {mem }}$ is generated according to Eq. (8) or (10).

\section{$<$ Retrieval Procedure $>$}

1. A target pattern $\xi_{\mu}^{\prime}$ is selected from $\mathcal{S}$.

2. The unfixed qubits in the key input $\xi_{\text {key }}$ are defined for retrieval.

3. $H_{\text {fix }}$ is generated to fix some qubits according to $\xi_{\text {key }}$ with Eq. (12).

4. The quantum state is changed following Eq. (16), with the adiabatic Hamiltonian evolution given by Eq. (14) or (15).

\section{Retrieval Performance of 4-Qubit QuAM}

We performed pattern recall simulations in order to evaluate the QuAM retrieval performance. The setup was almost identical to that for the numerical simulations for nominal estimation of the memory capacity discussed in Sect. 3.1.2. We composed a 4-qubit QuAM and executed all QuAM operations. The QuAM state evolved with $T=3000$ according to Eq. (16). The magnitude of $\Delta H$ was $O\left(10^{-2}\right)$, while those of $H_{\text {mem }}$ and $H_{\text {fix }}$ were $O\left(10^{0}\right)$. By measuring $|\Psi(T)\rangle$, we evaluated $\left\langle P_{\text {ret }}\right\rangle$, which is the average probability of retrieving a correct $\xi_{\mu}^{\prime}$ associated with $\xi_{\text {key }}$. In the following, we statistically analyze all cases of $\mathcal{S}$, i.e., we examine all combinations of $M$ patterns chosen from $2^{4}$ patterns.

$\left\langle P_{\text {ret }}^{\mathrm{IS}}\right\rangle$, which is $\left\langle P_{\text {ret }}\right\rangle$ obtained with $H_{\mathrm{mem}}^{\mathrm{IS}}$, is shown in Fig. 2. $\left\langle P_{\text {ret }}^{\mathrm{IS}}\right\rangle_{\mathrm{S}}$ with 1 and 2 unfixed qubits are shown in Figs. 2 (a) and (b), respectively. These results indicate that the QuAM performance degrades if the number of unfixed qubits increases. It is also apparent that $\left\langle P_{\text {ret }}^{\mathrm{IS}}\right\rangle$ declines along with $M$ in both cases. When $A_{\text {fix }}$ takes a non-zero value, the QuAM performance is improved. Note that $A_{\mathrm{fix}}=0$ indicates that $H_{\text {fix }}$ is not used, and corresponds to the random selection of one pattern among the $2 M$ memorized patterns. In fact, the $\left\langle P_{\text {ret }}^{\mathrm{IS}}\right\rangle_{\mathrm{s}}$ with $A_{\text {fix }}=0$ in Figs. 2 (a) and (b) meet $1 / 2 M$. Therefore, the results indicate the retrieval efficacy of $H_{\text {fix }}$. However, we note here that $\left\langle P_{\text {ret }}^{\mathrm{IS}}\right\rangle$ becomes saturated if $A_{\text {fix }}$ takes 0.1 or higher.

The upper limit of $\left\langle P_{\text {ret }}^{\mathrm{IS}}\right\rangle$ is caused by the conflict between the memorized patterns and their inverted patterns. For example, we consider the memorization of a set $\mathcal{S}^{\text {eg }}=\left\{\xi_{1}, \xi_{2}\right\}$, where $\xi_{1}=(-1,-1,-1,+1)^{\mathrm{T}}$ and $\xi_{2}=(-1,+1,+1,-1)^{\mathrm{T}}$. Thus, the QuAM also memorizes the inverted patterns $\bar{\xi}_{1}=(+1,+1,+1,-1)^{\mathrm{T}}$ and $\bar{\xi}_{2}=$ $(+1,-1,-1,+1)^{\mathrm{T}}$. These 4 patterns are realized as a superposition state in the ground state of $H_{\mathrm{mem}}$, and their probabilities are the same. If we choose $\xi_{1}$ as a target pattern and select the last qubit $y_{4}$ as an unfixed qubit, the key input is $\xi_{\text {key }}^{\text {eg1 }}=\left(-1,-1,-1, y_{\text {un }}\right)^{\mathrm{T}}$, where $y_{\text {un }}$ denotes an unfixed qubit. In this case, the QuAM successfully retrieves 


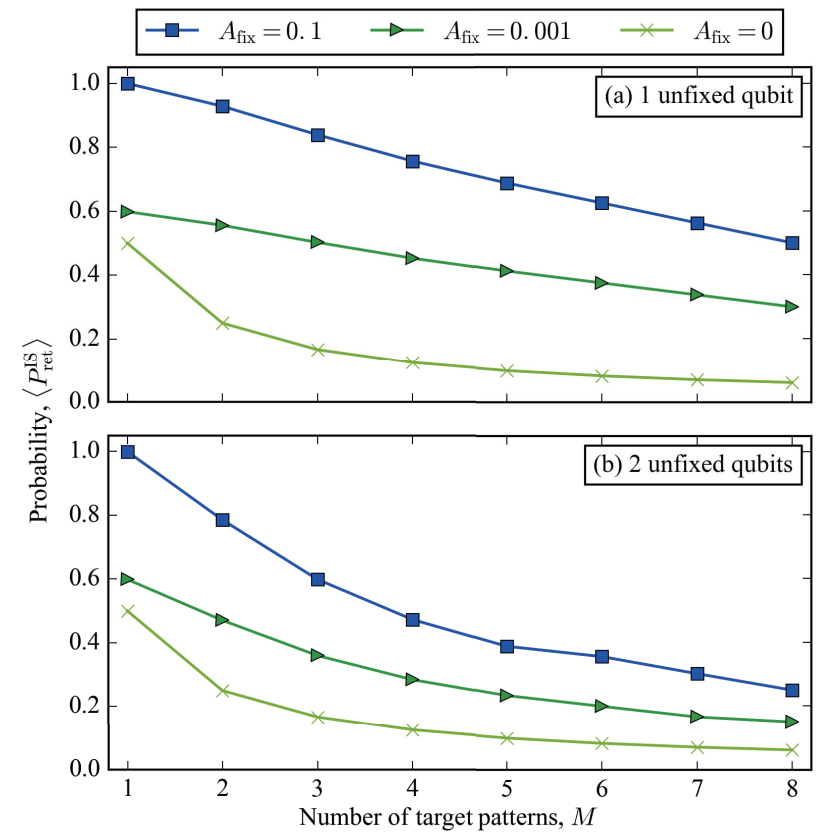

Fig. $2 A_{\text {fix }}$-dependency of retrieval probabilities of correct target pattern $\left\langle P_{\text {ret }}\right\rangle$ obtained with $H_{\mathrm{mem}}^{\mathrm{IS}}$. (a) $\left\langle P_{\mathrm{ret}}^{\mathrm{IS}}\right\rangle$ associated with a 1-unfixed-qubit key input and (b) $\left\langle P_{\text {ret }}^{\mathrm{IS}}\right\rangle$ associated with a 2-unfixed-qubit key input.

$\xi_{1}$, because there is no conflict. On the other hand, if we select the first qubit $y_{1}$ as an unfixed qubit, the key input is $\xi_{\mathrm{key}}^{\mathrm{eg} 2}=\left(y_{\mathrm{un}},-1,-1,+1\right)^{\mathrm{T}}$. In this case, the QuAM cannot distinguish $\xi_{1}$ from $\bar{\xi}_{2}$ according to $\xi_{\mathrm{key}}^{\mathrm{eg} 2}$, and the probabilities of $\xi_{1}$ and $\bar{\xi}_{2}$ take the same value (50\% each). In this way, the QuAM often fails to retrieve a target pattern, even if $H_{\text {fix }}$ succeeds in fixing the qubit states correctly. This inherent conflict of patterns occurs inevitably and its frequency increases with increasing $M$. The conflict occurs necessarily, especially in the case of $M=8$, because the QuAM memorizes all $2^{4}$ patterns. Thus, $\left\langle P_{\text {ret }}^{\mathrm{IS}}\right\rangle$ at $M=8$ should be $50 \%$. In the same manner, $\left\langle P_{\text {ret }}^{\mathrm{IS}}\right\rangle$ for the case of two unfixed qubits should be $25 \%$. In Fig. 2, the actual probabilities are obtained via simulation, as expected. Thus, we claim that our results are reasonable.

$\left\langle P_{\text {ret }}^{\mathrm{NI}}\right\rangle$, which is $P_{\text {ret }}$ obtained with $H_{\mathrm{mem}}^{\mathrm{NI}}$, is shown in Fig. 3. The difference between Figs. 3 (a) and (b) is the number of unfixed qubits. The $M$ - and $A_{\text {fix }}$-dependency of $\left\langle P_{\text {ret }}^{\mathrm{NI}}\right\rangle$ are also confirmed. Their tendencies are the same as for the case of QuAM with $H_{\mathrm{mem}}^{\mathrm{IS}}$, although its changes along with $M$ or $A_{\text {fix }}$ are partially non-monotonic. Unlike $H_{\text {mem }}^{\mathrm{IS}}$, $H_{\mathrm{mem}}^{\mathrm{NI}}$ memorizes patterns based on the interactions between two qubits. Because $H_{\mathrm{mem}}^{\mathrm{NI}}$ is composed of non-diagonal elements, its ground states are given as mixed states composed of desired and undesired states. Therefore, the QuAM with $H_{\mathrm{mem}}^{\mathrm{NI}}$ often retrieves fake patterns, which are irrelevant to the memorized and inverted patterns. However, the efficacy of using $H_{\mathrm{fix}}$ for the QuAM with $H_{\mathrm{mem}}^{\mathrm{NI}}$ is again confirmed.

At the end of this section, we discuss the difference between the $A_{\mathrm{fix}}$ values for the experiments using $H_{\mathrm{mem}}^{\mathrm{IS}}$ and $H_{\mathrm{mem}}^{\mathrm{NI}}$. The magnitude of $A_{\mathrm{fix}}$ in the case of $H_{\mathrm{mem}}^{\mathrm{IS}}$ is smaller

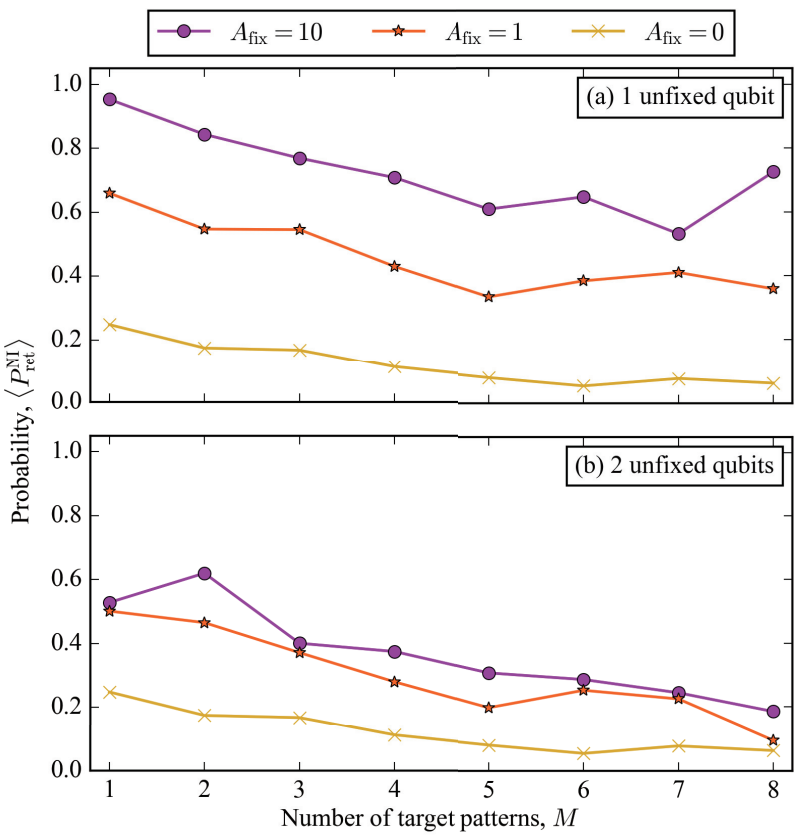

Fig. $3 A_{\text {fix }}$-dependency of retrieval probabilities of correct target pattern $\left\langle P_{\text {ret }}\right\rangle$ obtained with $H_{\mathrm{mem}}^{\mathrm{NI}}$. (a) $\left\langle P_{\text {ret }}^{\mathrm{NI}}\right\rangle$ associated with a 1-unfixed-qubit key input and (b) $\left\langle P_{\text {ret }}^{\mathrm{NI}}\right\rangle$ associated with a 2-unfixed-qubit key input.

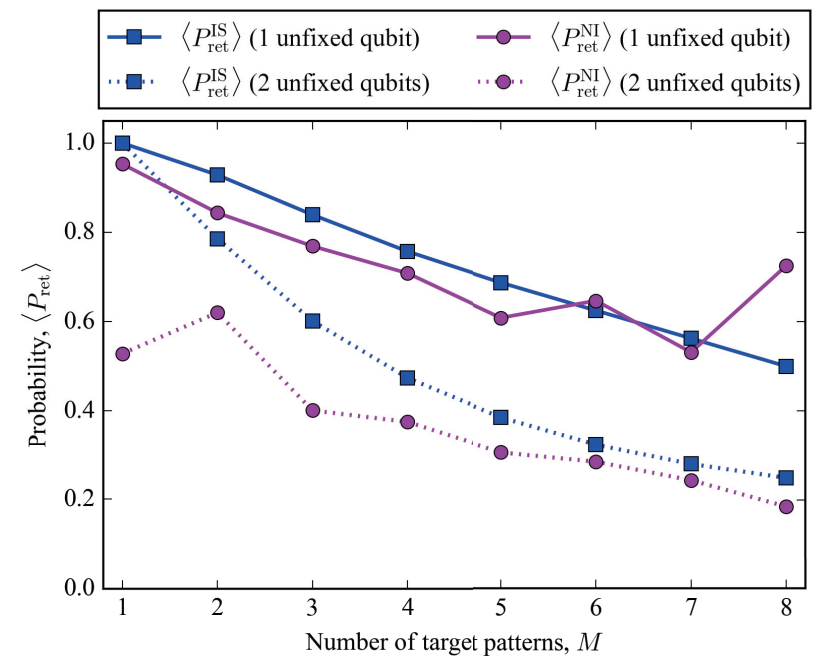

Fig. 4 Comparison between $\left\langle P_{\text {ret }}^{\mathrm{IS}}\right\rangle$ with $A_{\text {fix }}=0.1$ (blue lines) and $\left\langle P_{\text {ret }}^{\mathrm{NI}}\right\rangle$ with $A_{\mathrm{fix}}=10$ (purple lines). The solid and dotted lines denote the cases of 1 and 2 unfixed qubits, respectively.

than that for $H_{\mathrm{mem}}^{\mathrm{NI}}$. During the evolution, the QuAM amplifies the probability of one specific state according to $H_{\text {fix }}$ and retrieves a correct target pattern. $A_{\text {fix }}$ determines the strength of the qubit state fixing and controls the degree of amplification. Because the ground states of $H_{\mathrm{mem}}^{\mathrm{NI}}$ are given as mixed states, the energy difference between the target pattern and the other patterns is small, and thus, a relatively large $A_{\mathrm{fix}}$ is required to definitely retrieve the correct pattern. Sufficient $\left\langle P_{\text {ret }}^{\mathrm{NI}}\right\rangle$ is obtained with $A_{\mathrm{fix}}=10$. In Fig. 4, we compare two $\left\langle P_{\text {ret }}\right\rangle$ s; $\left\langle P_{\text {ret }}^{\mathrm{IS}}\right\rangle$ with $A_{\text {fix }}=0.1$ (Fig. 2) and $\left\langle P_{\text {ret }}^{\mathrm{NI}}\right\rangle$ with $A_{\text {fix }}=10$ (Fig. 3). When $M$ is small, $\left\langle P_{\text {ret }}^{\mathrm{IS}}\right\rangle$ (blue line 
with squares) is higher than $\left\langle P_{\text {ret }}^{\mathrm{NI}}\right\rangle$ (purple line with circles), and this difference is clearly confirmed when the number of unfixed qubits is 2 (dotted lines). However, although this difference exists, these two types of QuAM exhibit almost the same performance overall. Thus far, we have evaluated the retrieval performance and confirmed that our QuAM can memorize multiple patterns and can retrieve one specific pattern associated with a given $\xi_{\text {key }}$. Because the $M_{\text {cap }}$ of the Hopfield network is limited to $0.14 n$ [25], where $n$ is the number of neurons, our QuAM model exhibits superior performance, if we allow some retrieval errors.

\section{Conclusion}

We have proposed a new quantum associative memory (QuAM) model with a quantum neural network by relating neural and quantum computing. The association function is realized via adiabatic Hamiltonian evolution, and the Hamiltonians are composed of $H_{\mathrm{mem}}$ for pattern memorization and $H_{\text {fix }}$ to provide a key input. We studied two types of $H_{\mathrm{mem}}$, i.e., an Ising Hamiltonian $H_{\mathrm{mem}}^{\mathrm{IS}}$ used for quantum annealing and a neuro-inspired Hamiltonian $H_{\mathrm{mem}}^{\mathrm{NI}}$ used for neuromorphic adiabatic quantum computation. Numerical simulation results indicate that the proposed memorization and retrieval operations work properly with both types of Hamiltonian. Because their performances are very similar, one should select a Hamiltonian for pattern memorization based on the hardware feasibility. Surprisingly, the QuAM can memorize $2^{N-1}$ patterns at once because of its quantum mechanical property; however, it cannot always successfully retrieve one specific target pattern because of the inherent conflicts between the memorized and inverted patterns. Although some errors in the retrieval process are inevitable, our QuAM exhibits new and unique features that differ from a conventional neural associative memory.

\section{Acknowledgments}

This work was partially supported by JSPS KAKENHI Grant Number 15K18044 and Okawa Foundation. We would like to thank Editage (www.editage.jp) for English language editing.

\section{References}

[1] M.W. Johnson, M.H.S. Amin, S. Gildert, T. Lanting, F. Hamze, N. Dickson, R. Harris, A.J. Berkley, J. Johansson, P. Bunyk, E.M. Chapple, C. Enderud, J.P. Hilton, K. Karimi, E. Ladizinsky, N. Ladizinsky, T. Oh, I. Perminov, C. Rich, M.C. Thom, E. Tolkacheva, C.J.S. Truncik, S. Uchaikin, J. Wang, B. Wilson, and G. Rose, "Quantum annealing with manufactured spins," Nature, vol.473, no.7346, pp.194-198, 2011.

[2] C.C. McGeoch and C. Wang, "Experimental evaluation of an adiabiatic quantum system for combinatorial optimization," Proc. ACM International Conference on Computing Frontiers, p.23, ACM, 2013.

[3] T. Kadowaki and H. Nishimori, "Quantum annealing in the transverse ising model," Phys. Revi. E, vol.58, no.5, p.5355, 1998

[4] S. Kirkpatrick, C.D. Gelatt, and M.P. Vecchi, "Optimization by simulated annealing," Science, vol.220, no.4598, pp.671-680, 1983.
[5] V.S. Denchev, S. Boixo, S.V. Isakov, N. Ding, R. Babbush, V. Smelyanskiy, J. Martinis, and H. Neven, "What is the computational value of finite-range tunneling?," Phys. Revi. X, vol.6, no.3, p.031015, 2016.

[6] P.W. Shor, "Polynomial-time algorithms for prime factorization and discrete logarithms on a quantum computer," SIAM review, vol.41, no.2, pp.303-332, 1999.

[7] L.K. Grover, "A fast quantum mechanical algorithm for database search," Proc. twenty-eighth annual ACM symposium on Theory of computing, pp.212-219, ACM, 1996.

[8] J. Schmidhuber, "Deep learning in neural networks: An overview," Neural Networks, vol.61, pp.85-117, 2015.

[9] S. Kak, "On quantum neural computing," Information Sciences, vol.83, no.3, pp.143-160, 1995.

[10] M.V. Altaisky, N.E. Kaputkina, and V.A. Krylov, "Quantum neural networks: Current status and prospects for development," Physics of Particles and Nuclei, vol.45, no.6, pp.1013-1032, 2014.

[11] A. Manju and M.J. Nigam, "Applications of quantum inspired computational intelligence: a survey," Artificial Intelligence Review, vol.42, no.1, pp.79-156, 2014.

[12] K.H. Han and J.H. Kim, "Quantum-inspired evolutionary algorithm for a class of combinatorial optimization," IEEE Trans. Evol. Comput., vol.6, no.6, pp.580-593, Dec. 2002.

[13] M. Fiasché, A Quantum-Inspired Evolutionary Algorithm for Optimization Numerical Problems, pp.686-693, Springer Berlin Heidelberg, 2012.

[14] S. Sato, M. Kinjo, and K. Nakajima, "An approach for quantum computing using adiabatic evolution algorithm," Jpn. J. Appl. Phys., vol.42, no.11R, p.7169, 2003.

[15] E. Farhi, J. Goldstone, S. Gutmann, J. Lapan, A. Lundgren, and D. Preda, "A quantum adiabatic evolution algorithm applied to random instances of an np-complete problem," Science, vol.292, no.5516, pp.472-475, 2001.

[16] M. Kinjo, S. Sato, Y. Nakamiya, and K. Nakajima, "Neuromorphic quantum computation with energy dissipation," Phys. Revi. A, vol.72, no.5, p.052328, 2005.

[17] Y. Osakabe, S. Sato, M. Kinjo, K. Nakajima, H. Akima, and M. Sakuraba, "Learning method for a quantum bit network," Lect. Notes Comput. Sci., Artificial Neural Networks and Machine Learning - ICANN 2016: 25th International Conference on Artificial Neural Networks, p.558, Springer, 2016.

[18] D. Ventura and T. Martinez, "Quantum associative memory," Information Sciences, vol.124, no.1, pp.273-296, 2000.

[19] M. Peruš and S. Dey, "Quantum systems can realize contentaddressable associative memory," Applied mathematics letters, vol.13, no.8, pp.31-36, 2000.

[20] R. Allauddin, S. Boehmer, E.C. Behrman, K. Gaddam, and J.E. Steck, "Quantum Simulataneous Recurrent Networks for Content Addressable Memory," pp.57-76, Springer Berlin Heidelberg, 2008.

[21] A. Messiah, "Quantum mechanics, two volumes," Dover Publications, 1999.

[22] J.J. Hopfield, "Neural networks and physical systems with emergent collective computational abilities," Proc. National Academy of Sciences, vol.79, no.8, pp.2554-2558, 1982.

[23] S. Morita and H. Nishimori, "Mathematical foundation of quantum annealing," J. Mathematical Physics, vol.49, no.12, p.125210, 2008.

[24] C.F. Van Loan, Matrix computations (Johns Hopkins studies in mathematical sciences), The Johns Hopkins University Press, 1996.

[25] S.I. Amari and K. Maginu, "Statistical neurodynamics of associative memory," Neural Networks, vol.1, no.1, pp.63-73, 1988. 


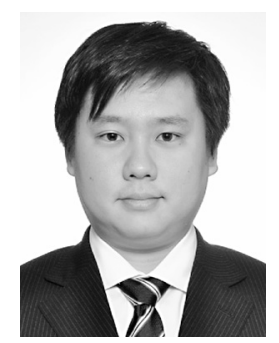

Yoshihiro Osakabe received the B.E., M.E. degrees from Tohoku University, Sendai, Japan, in 2013 and 2015, respectively. Currently, he is a Ph.D. student at Tohoku University, Sendai Japan. His research interests include quantum computing, especially quantum algorithms and its hardware implementation. He is a student member of IEEE.

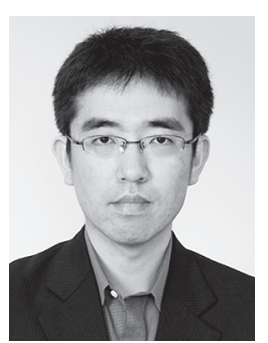

Hisanao Akima received the B.E., M.S., and $\mathrm{Ph} . \mathrm{D}$. degrees from Tohoku University, Sendai, Japan, in 2000, 2002, and 2006, respectively. From 2006 to 2013, He was with the Hitachi, Ltd., Central Research Laboratory, Tokyo, Japan, where he worked on spherical aberration corrector for electron microscope. In 2013, he joined the Research Institute of Electrical Communication, Tohoku University. His current research interests include neuromorphic hardware, visual information processing, and brainlike very large scale integrated (VLSI) circuit.

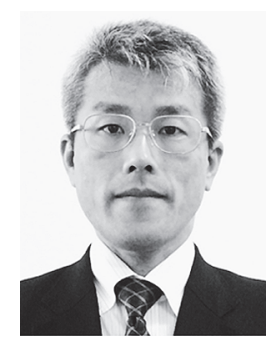

Masao Sakuraba received the B.E., M.E. and Ph.D. degrees from Tohoku University in 1990, 1992, and 1995, respectively. There, he has been engaged in the development of atomic layer growth control of germanium and silicon and research on surface reaction mechanisms. He became a Research Associate and an Associate Professor of Tohoku University in 1995 and 2002, respectively. Now, in the Laboratory for Nanoelectronics and Spintronics of Research Institute of Electrical Communication, he is actively involved in the development of low-damage plasma chemical-vapor deposition processing for group IV quantum heterointegration.

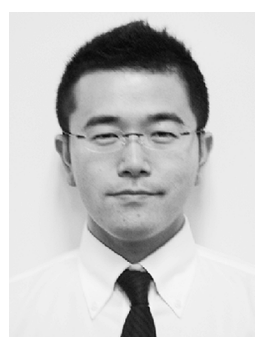

Mitsunaga Kinjo received B.E. degree in Electrical and Information Engineering from University of the Ryukyus, Okinawa, Japan in 1996. And he received M.E. degree in Electrical Engineering and Ph.D. degree in Information Science from Tohoku University, Sendai, Japan in 1998 and 2001, respectively. From April 2001 to September 2003, he was a researcher in the PRESTO program of the Japan Science and Technology Corporation (JST) conducted by Dr. Shigeo SATO at Tohoku University. From October 2003 to September 2006, he was a research fellow of the Research Institute of Electrical Communication (RIEC), Tohoku University. He worked on the development of quantum computation algorithm and its hardware implementation. Currently he is an associate professor in the Faculty of Engineering, University of the Ryukyus, since October 2008. His research interests are in quantum computation and its hardware implementation.

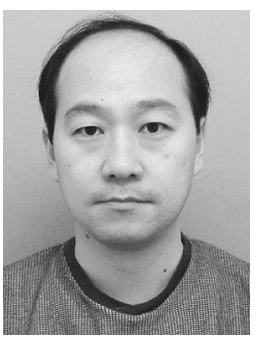

Shigeo Sato received the B.S., M.S., and Ph.D. degrees from Tohoku University, Sendai, Japan, in 1989, 1991, and 1994, respectively. In 1996, he joined the Research Institute of Electrical Communication, Tohoku University. His research interests are in LSI implementation and application of neural networks. 